\title{
Cholecalciferol supplementation alters gut function and improves digestibility in an underground inhabitant, the naked mole rat (Heterocephalus glaber), when fed on a carrot diet
}

\author{
BY SHLOMO YAHAV* \\ MRC Mineral Metabolism Research Unit, Department of Paediatrics, University of the \\ Witwatersrand, Baragwanath Hospital, Johannesburg, PO Bertsham 2013, South Africa \\ AND ROCHELLE BUFFENSTEIN $\dagger$ \\ Physiology Department, University of the Witwatersrand, Medical School, 7 York Road, Parktown, \\ Johannesburg 2193, South Africa
}

(Received 18 February 1991-Accepted 21 February 1992)

\begin{abstract}
Naked mole rats (Heterocephalus glaber) lead a strictly subterranean existence and appear to be naturally deficient in cholecalciferol $\left(D_{3}\right)$. Oral supplementation with $D_{3}\left(D_{s}\right)$ led to a 18-fold increase in food intake and the associated enlargement (1-4-fold) of the caecum. The effect of $D_{s}$, and the concomitant increase in food intake, on caecal fermentation efficiency when animals were fed on a carrotbased diet was determined by measuring the rate of both gas production and short-chain fatty acid (SCFA) production. Microbial-controlled fermentation processes in the caecum were enhanced with $D_{s}$ when compared with animals not receiving a $D_{3}$ supplement $\left(D_{n}\right)$. Both the rates of gas production $\left(D_{n} 10.76\right.$ (SE 0.77), $D_{s} 15.20$ (SE 1.77) $\mathrm{ml} / \mathrm{g}$ dry matter (DM) per h) and SCFA production $\left(D_{n} 463.0\right.$ (SE 33.7), $D_{s} 684 \cdot 3$ (SE 74.8) $\mu \mathrm{mol} / \mathrm{g}$ DM per $\mathrm{h}$ ) increased more than 1.4-fold per $\mathrm{g}$ DM caecal substrate. These factors contributed to the higher digestibility of the food in $D_{s}$ animals. The larger quantity of energy available to $D_{3}$-replete naked mole rats was not used in anabolic processes, for these animals maintained mass. These findings suggest that metabolic rate in $D_{3}$-replete animals was elevated. Thus, despite improved gut function, $D_{3}$-replete animals may be disadvantaged by their higher energy and food requirements in their natural milieu.
\end{abstract}

Cholecalciferol: Caecal fermentation: Mole rats

The naked mole rat (Heterocephalus glaber; Rodentia; Bathyergidae) lives in an extensive maze of underground burrows in the compacted soils of arid north coast Africa. Here it feeds on a herbivorous diet of roots and tubers (Jarvis \& Bennett, 1991) which are scarce, randomly distributed and invariably high in fibre. Digestion of fibre is facilitated by a large population of symbiotic bacteria and protozoa in the hind-gut (Buffenstein \& Yahav, $1991 \mathrm{a}$ ). These micro-organisms digest the insoluble plant fibres, via fermentation processes, and in so doing produce gaseous waste products $\left(\mathrm{CO}_{2}, \mathrm{CH}_{4}\right.$ and $\left.\mathrm{H}_{2}\right)$ and short-chain fatty acids (SCFA). The SCFA (i.e. acetic, propionic, butyric acids) are absorbed by the host animal through the caecum and large intestine and are used as an energy source by the host animal (Rechkemmer et al. 1988).

Fermentation processes contribute to the digestibility and maximal usage of the available food. Maximal utilization of food is highly advantageous in an underground environment,

\footnotetext{
* Present address: The Volcani Institute of Animal Science, PO Box 6, Bet Dagan, 50250, Israel.

$\dagger$ For reprints.
} 
where there is a high energetic cost associated with the location of randomly distributed underground food supplies (Vleck, 1981).

Mole rats, living in an environment devoid of sunlight and consuming only plant material, appear to be naturally cholecalciferol $\left(\mathrm{D}_{3}\right)$-deficient. Serum $25-\mathrm{OH}$ vitamin $\mathrm{D}_{3}$ levels are undetectable $(<0.004 \mathrm{nmol} / \mathrm{ml} ;$ Buffenstein et al. 1988, 1991). In addition mole rat mineral absorption when fed on a diet of sweet potato (Ipomoea batatas Poir) and apple appears to be independent of $\mathrm{D}_{3}$ (Buffenstein \& Yahav, $1991 \mathrm{~b}$ ) and Ca absorption is via non-saturable passive processes (Skinner et al. 1991; Buffenstein \& Yahav, 1991 b). Mole rats, however, can metabolize tritium-labelled $\mathrm{D}_{3}$ to its more polar metabolites and possess calbindins thought to be $\mathrm{D}_{3}$-dependent (Buffenstein et al. 1988). These findings imply that they do not normally require $D_{3}$ for mineral metabolism but may employ $D_{3}$, if and when it becomes available, in other physiological processes.

Deficiency in $\mathrm{D}_{3}$ is a complex nutritional state in which most mammals consume less (Chertow et al. 1983). A decrease in food intake might influence gut function. We, therefore, investigated the effect of an oral supplement of $\mathrm{D}_{3}$ on food intake and gut function in these chthonic naturally $\mathrm{D}_{3}$-deficient rodents when maintained on a carrotbased diet.

In captivity, mole rats, when given a variety of foods, select carrots, sweet potato and apple (Jarvis, 1991). Previous fermentation studies (Buffenstein \& Yahav, 1991a) have indicated that fermentation efficiency on a carrot diet was superior to that on sweet potato and apple. Furthermore, we have previously found that $\mathrm{D}_{3}$ supplementation had no effect on the damara mole rat (Cryptomys damarensis) when fed on sweet potato and apple (Skinner et al. 1991), whereas it had a pronounced effect when animals were fed on carrots (unpublished results). We, therefore, examined whether fermentation efficiency improved with $\mathrm{D}_{3}$ supplementation.

\section{MATERIALS AND METHODS}

\section{Animals and treatment}

Non-breeding, adult (1-2-year-old) male naked mole rats were used in the present study. These animals were born in captivity. The progenitors of these animals were collected in Kenya. Captive mole rats were housed in climatically controlled rooms $\left(30^{\circ} \pm 2^{\circ} ; 75 \%\right.$ relative humidity) in accordance with the housing protocol suggested by Jarvis (1991) for a minimum period of 6 months before experimentation. These rooms had no access to natural light and animals were housed under $\operatorname{dim}(20 \mathrm{~W})$ incandescent light. Mole rats show no circadian rhythm in activity and time of feeding and were, therefore, always supplied ad $l i b$. with carrots. They were maintained on this diet for 6 weeks before experimentation.

At $10 \mathrm{~d}$ before monitoring food intake and excretory losses, animals were housed individually in metabolism cages and given free access to the chosen diet. Two groups of mole rats were used in the present study. Both were fed on the same fresh carrot diet and subjected to the same experimental conditions, the only difference being that an oral supplement of $D_{3}$ was given to the second group $\left(D_{s}\right)$.

The $\mathrm{D}_{3}$ dose was determined from the American Institute of Nutrition (1977) prescribed dose for rats $\left(25 \mathrm{ng} \mathrm{D}_{3} / \mathrm{g}\right.$ dry matter food eaten). This was administered orally in an oil vehicle $(0.1 \mathrm{mmol} / \mathrm{l})$ every $3 \mathrm{~d}$ during the experimental period, starting $6 \mathrm{~d}$ before the commencement of monitoring food intake and faecal output.

\section{Food budget}

Over a $12 \mathrm{~d}$ period, body mass, food supplied, left-over food and the quantity of faeces produced were monitored daily. Uneaten food, daily faecal output and representative food samples, dried to constant mass at $70^{\circ}$, were weighed. 


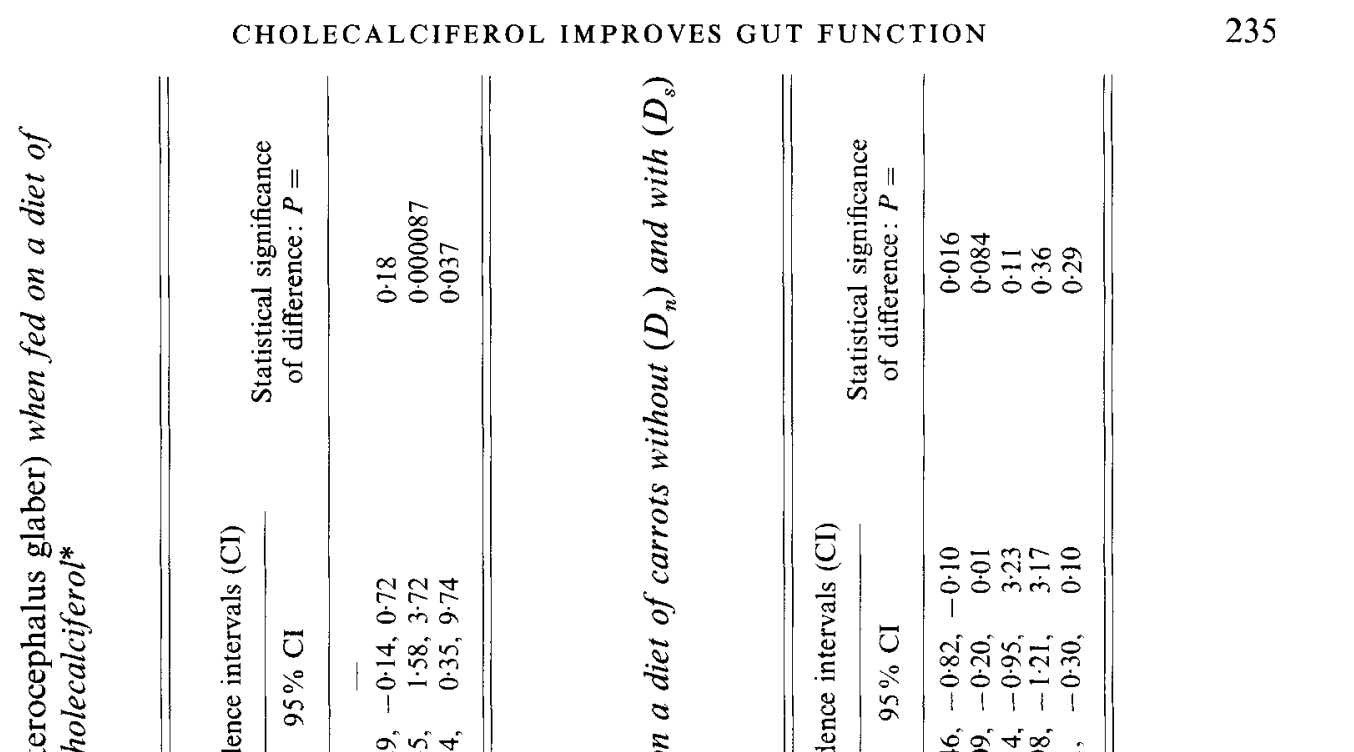




\section{Caecal measurements}

At the end of the period of 'food budget' monitoring, unstarved animals were killed, between 08.00 and 10.00 hours, by injecting Euthenase (Centaur Laboratory, Johannesburg) intraperitoneally (200 mg sodium pentobarbitone $/ \mathrm{ml} ; 0.01 \mathrm{ml} / \mathrm{g}$ body weight). The gastrointestinal tract was immediately removed intact, and the caecum separated from the rest of the gut. This was weighed and the $\mathrm{pH}$ measured. Thereafter the caecal contents were mixed to obtain uniform portions. Weighed portions (approximately $0.3 \mathrm{~g}$ ) were immediately used in fermentation studies and in the determination of dry matter content.

\section{Fermentation capacity}

Rates of gas production and short-chain fatty acid (SCFA) production were measured to determine fermentation capacity. This was done by transferring the weighed caecal sample to a $25 \mathrm{ml}$ Warburg flask and rapidly displacing the air within the flask with $\mathrm{CO}_{2}$. The manometric apparatus was kept open, allowing the displacement of $\mathrm{CO}_{2}$ by the gases produced during fermentation. Thereafter the flasks were sealed and incubated at $33^{\circ}$, the optimal temperature for caecal function in these animals (Yahav \& Buffenstein, 1991). The volume of gas produced in the flasks was monitored every $5 \mathrm{~min}$ for $1 \mathrm{~h}$ using standard manometric techniques. The gas was released after each period of monitoring. The rate of gas production at the time the caecum was excised was extrapolated from the exponential relationship between gas production and time, in compliance with the methods of Carol \& Hungate (1954).

Rate of SCFA production was determined from SCFA concentration in caecal samples taken after excising the caecum and those taken after the completion of gas production measurements (McBee, 1970). Known amounts of $\mathrm{NaOH}(100 \mathrm{~g} / \mathrm{l})$ were added to each sample. These samples were then stored at $-20^{\circ}$ for later analysis. The concentration of SCFA was determined using gas-liquid chromatography (Carlo Erba Strumentzione 4200, Searle Instruments, Johannesburg) using pivalic acid as an internal standard (Davis, 1988).

The dry matter content was determined by taking weighed portions of caecum content and drying these at $70^{\circ}$ to constant mass.

\section{Micro-organism analysis}

Bacteria and protozoa present in the caecal fluid were counted directly using a haemocytometer $(0 \cdot 1 \mathrm{~mm}$ deep) and light microscopy.

\section{Statistical analyses}

Untransformed data were subjected to a Kolmogorov-Smirnov test and the data were found to follow a normal distribution. Thereafter data from the two experimental treatments (with $\left(D_{s}\right)$ and without $\left(D_{n}\right) D_{3}$ supplementation) were compared using a twotailed unpaired Student's $t$ test (Zar, 1974). All values were expressed as means with their standard errors and were considered significantly different at $P \leqslant 0.05$.

\section{RESULTS}

Body mass

Irrespective of experimental treatment, animal weight remained relatively constant throughout the monitored experimental period (Table 1) and percentage change in mass between the two experimental treatments was not significant $(P=0 \cdot 18)$. 


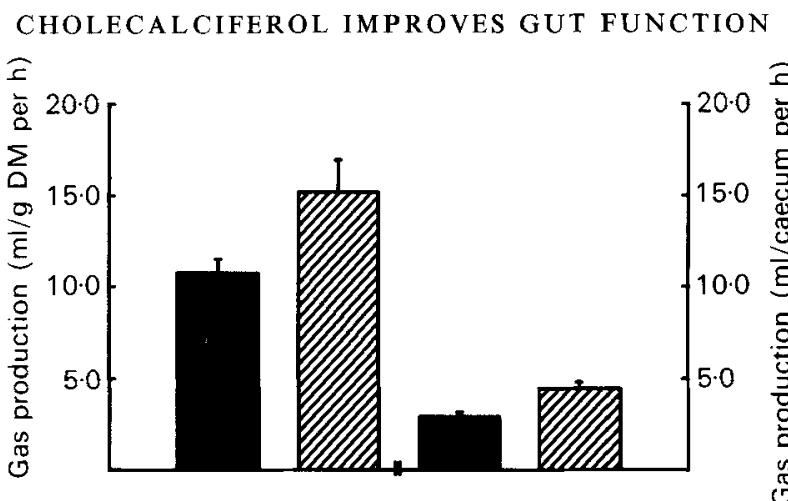

Fig. 1. The effect of cholecalciferol $\left(D_{3}\right)$ on rates of gas production (measured as zero gas production; see p. 236) in the naked mole rat (Heterocephalus glaber). Values are means with their standard errors represented by vertical bars. Mean values were significantly increased with cholecalciferol $\left(D_{3}\right)$ supplementation $(\theta)$ when compared with that of animals not receiving an oral $\mathrm{D}_{3}$ supplement $(\square)$ : on $\mathrm{ml} / \mathrm{g}$ dry matter $(\mathrm{DM})$ per $\mathrm{h}$ basis $P=0.031$; on a $\mathrm{ml} /$ caecum per h basis $P=0.028$.

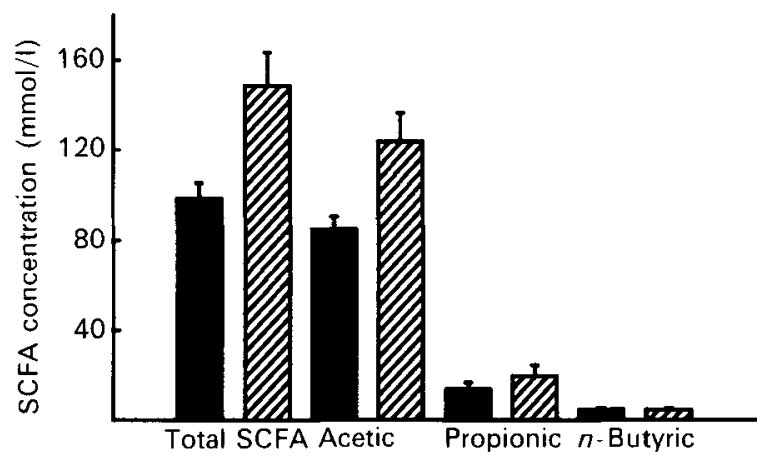

Fig. 2. Initial short-chain fatty acid (SCFA) concentrations in the caecum of the naked mole rat (Heterocephalus glaber) when fed on a diet of carrots without $(\square)$ and with $(\mathbb{C})$ an oral cholecalciferol $\left(\mathrm{D}_{3}\right)$ supplement. Values are means with their standard errors represented by vertical bars. Mean values were significantly different for total SCFA and acetic acid $P=0.0061$. All other pairs of values were not significantly different. For details of procedures, see pp. 234-236.

\section{Food intake}

Oral supplementation of $\mathrm{D}_{3}$ had a pronounced effect on appetite. Food intake increased 1.8fold (Table 1). Increase in food intake was accompanied by a $5 \cdot 1$ percentage units increase $(P=0.037)$ in digestibility.

\section{Caecal measurements}

Caecal mass (expressed relative to final mass, per $100 \mathrm{~g}$ animal) increased 1.4-fold with $\mathrm{D}_{3}$ supplementation (Table 2 ). Caecal wall mass increased $1 \cdot 2$-fold; however, this trend was not statistically confirmed $(P>0 \cdot 05)$. Supplementation with $\mathrm{D}_{3}$ had no effect on caecal $\mathrm{pH}$ (Table 2).

The average number of protozoa $(1 \cdot 4$-fold $)$ and bacteria $(1 \cdot 1$-fold $)$ in caecal fluid increased with $\mathrm{D}_{\mathrm{s}}$. However, these trends were not statistically confirmed $(P \geqslant 0 \cdot 10$; Table 2).

\section{Gas production}

Gas production per g dry matter increased 1.4-fold $(P \leqslant 0.03)$ with $\mathrm{D}_{\mathrm{s}}$. When the enlarged caecal mass is taken into account gas production per caecum increased 1.53 -fold $(P=0.03)$ with $\mathrm{D}_{\mathrm{s}}$ (Fig. 1). 


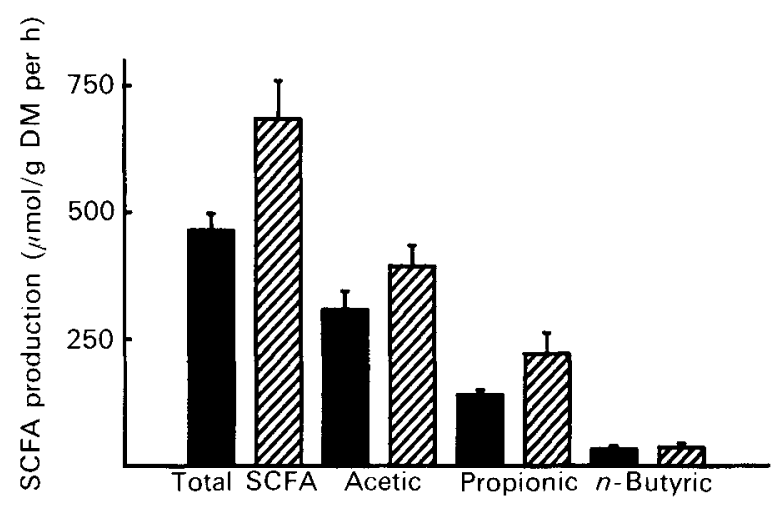

Fig. 3. Rates of short-chain fatty acid (SCFA) production (/g dry matter) in the naked mole rat (Heterocephalus glaber) when fed on a diet of carrots without $(\square)$ and with $(\square)$ an oral cholecalciferol $\left(D_{3}\right)$ supplement. Values are means with their standard errors represented by vertical bars. Mean values for total SCFA were significantly different: $P=0.03$. All other pairs of values were not significantly different. For details of procedures, see pp. $234-236$.

\section{$S C F A$}

Oral supplementation of $\mathrm{D}_{3}$ resulted in a significant increase in total SCFA concentration and acetic acid concentration $(P \leqslant 0.0061$ and $P \leqslant 0.0128$ respectively) in the caecum. When values were expressed per $g$ dry matter, a significant increase in the total SCFA production was demonstrated in $\mathrm{D}_{\mathrm{s}}$ animals (Fig. 2). The values for SCFA produced in $\mathrm{D}_{3}$ replete animals:SCFA in deficient animals were $1.27 ; 1.58$; and 1.11 for acetic, propionic and butyric acids respectively. The increases in production rate of individual SCFA were not statistically significant. When the individual increments of each SCFA were compounded, however, total SCFA production was significantly enhanced with $\mathrm{D}_{s}$ (Figs. 2 and 3$)$.

\section{DISCUSSION}

Despite the fact that naked mole rats appear to be naturally $\mathrm{D}_{3}$ deficient (Buffenstein et al. 1988), oral supplementation of $D_{3}$ led to an increase in appetite, food digestibility and a marked improvement in caecal function.

Food intake followed reported trends (Chertow et al. 1983; Kadowaki \& Norman, 1984; Nyomba et al. 1984; Clark et al. 1986) and increased 1.8 times with $\mathrm{D}_{\mathrm{s}}$ (Table 1). This could be attributed to either a direct effect on appetite receptors in hypothalamus (Stumpf et al. 1982) or an indirect effect via changes in carbohydrate metabolism. Carbohydrate metabolism in $\mathrm{D}_{3}$-deficient animals is known to be defective (Norman et al.1980; Clarke et al. 1981). Insulin secretion is particularly affected, in that it is impaired in $\mathrm{D}_{3}$-deficient animals and enhanced by $\mathrm{D}_{3}$ supplementation (Norman et al. 1980; Kadowaki \& Norman, 1984; Clark et al. 1986). Changes in blood glucose levels with $D_{3}$ supplementation, may in turn lead to an increase in appetite and food intake.

Naked mole rats showed no response to $\mathrm{D}_{3}$ supplementation when fed on a diet of sweet potato and apple (Buffenstein \& Yahav, 1991b), yet when the diet was altered to that of carrot (in the present study), appetite was enhanced. Differences between these two studies could possibly be attributed to different dietary fibre, glucose and starch contents (Buffenstein \& Yahav, 1991 a).

Despite the pronounced increase in food intake and digestibility, naked mole rats showed no significant increase in body mass (Table 1). These findings suggest that energy expenditure was raised in response to $\mathrm{D}_{3}$ supplementation. Clark et al. (1986) showed 
similar mass constancy despite an increase in food intake and attribute this to increased soft tissue metabolism. Increased food requirements and elevated metabolism in response to $D_{3}$ supplementation would disadvantage animals living underground in an environment where food resources are limiting and their underground location is energetically costly (Vleck, 1981). Furthermore an elevation in metabolic rate, with the concomitant increase in heat production, would handicap the ability of these underground inhabitants of warm equatorial Africa to keep body temperature below lethal levels. Mechanisms of heat loss in an underground closed environment are impeded by the high relative humidities which preclude the efficient use of evaporative cooling and also by the absence of air movement for convective cooling. Naked mole rats naturally exhibit a reduced metabolic rate when compared with superterranean rodents and this is thought to be highly adaptive to the prevailing burrow conditions (Buffenstein \& Yahav, 1991c).

In most hind-gut fermenters, increased food intake is accompanied by a decline in food digestibility (Sibly, 1981; Van Soest, 1982). Mole rats did not conform with this generalization; instead, digestibility improved (Table 1). Increased food intake and digestibility were accompanied by an enlargement in caecal size (Table 2). Similar improvements in food utilization with increased food intake have been reported when $D_{3}$ deficient rats were given $D_{3}$ supplementation (Clark et al. 1986). The mode of action of $D_{3}$ supplementation on digestibility is, however, not known. We suggest that improved digestibility is a result of enhanced microbial-controlled fermentation processes in the enlarged caecum. The precise mechanism is unknown. We speculate that the number and efficacy of micro-organisms increases when an oral $\mathrm{D}_{3}$ supplementation is given.

Evidence supporting an improvement in microbial-controlled fermentation processes with $\mathrm{D}_{\mathrm{s}}$ include a 1.4-fold increase in both the rate of gas production (Fig. 1) and the total SCFA production per g substrate dry matter (Fig. 2). Substrate utilization and microbial functional efficiency were, thus, enhanced. Fermentative micro-organisms can metabolize $\mathrm{D}_{3}$, albeit to unusual derivatives (Sommerfeldt et al. 1980; Gardener et al. 1988), and it is possible that $D_{3}$ directly affects microbial function The mode of action of $D_{3}$ on microbial function, however, has to date not been investigated.

Population counts on micro-organisms in the fermentative chamber (caecum/rumen) fluid may only serve as indicators of the true population density. This is because most of the fermentative micro-organisms adhere to plant fibres and are excluded from population assessments (El-Din \& El-Shazly, 1969). Trends (whilst not statistically significant) in population density in the caecal fluid showed that the number of bacteria increased with $\mathrm{D}_{\mathrm{s}}$ at least 1-1-fold and that the number of protozoa increased 1-4-fold (Table 2). Similar incremental changes (1.4-1.5-fold) in the number of protozoa, gas and total SCFA production per $g$ dry matter suggest that $D_{3}$ had a direct effect on microbial proliferation and that the improved microbial function (per g substrate dry matter) is a reflection of, primarily, the change in protozoa population density. Not only does the number of microorganisms appear to increase with $\mathrm{D}_{\mathrm{s}}$, but protozoal species diversity also appears enhanced. In the absence of a $\mathrm{D}_{3}$ supplement, only one bi-flagellate protozoal species was seen (Buffenstein \& Yahav, 1991a). At least three other holotrich ciliate species were observed in caecal fluid samples taken from $D_{s}$ animals ( $S$. Yahav \& R. Buffenstein, unpublished results). The different values for SCFA produced in $D_{s}: S C F A$ in $D_{n}$ for the three acids (acetic 1.26; propionic 1.58; butyric 1.11) suggest that increased SCFA production is not merely due to an increase in population density but may be also due to species changes with $D_{s}$.

Enhanced fermentation efficiency coupled with the 1.4-fold increase in caecal mass elicited the doubling of the total SCFA available to the naked mole rat (Figs. 2 and 3). These SCFA are an important energy source to the host animal (Van Soest, 1982) as they 
are absorbed into the blood and provided an energy-rich substrate for metabolism (Rechkemmer et al. 1988). Oral supplementation of $\mathrm{D}_{3}$ assists in the liberation of a large energy supply that would otherwise be lost to this underground inhabitant. The increase in available energy is, however, not used in anabolic processes by these animals and appears rather to be dissipated by an elevation in metabolic rate.

In conclusion, hind-gut microbial function is enhanced by oral $\mathrm{D}_{3}$ supplementation. This results in improved food utilization and a concomitant larger energy supply to the host animal. The additional energy is dissipated, with no evidence for anabolic usage, as these animals maintained body mass despite a 1.8-fold increase in food intake by $\mathrm{D}_{3}$-replete mole rats. As $\mathrm{D}_{3}$ repletion is not essential for mineral homeostasis (Buffenstein \& Yahav, $1991 b$ ), a $\mathrm{D}_{3}$-replete status does not afford any obvious advantage to these underground inhabitants.

The authors wish to thank Professor Jenny Jarvis for providing the animals used in this study. These animals were housed in the Central Animal Service of the University of the Witwatersrand and the staff of the Central Animal Unit are thanked for their assistance in caring for them. The foods used in this study were analysed by Ms Joan Bernan of the Nutritional Laboratory at the Animal and Dairy Science Research Institute. Mr Jock Davies and Ms Joan Bernan kindly enabled us to use the equipment at the Animal and Dairy Research Institute at Irene, Transvaal. This work was supported by a CSIR/FRD grant and approved by the Animal Ethics Committee of the University of the Witwatersrand (no. 89/34/2).

\section{REFERENCES}

American Institute of Nutrition (1977). Report of the A.I.N. ad hoc committee on standards for nutritional studies. Journal of Nutrition 107, 1340.

Buffenstein, R., Jarvis, J. U. M., Opperman, L. A., Cavaleros, M., Hough, S. \& Ross, P. (1988). Vitamin D metabolism and expression in chthonic naked mole rats. Journal of Bone and Mineral Research 3, S119 Abstr.

Buffenstein, R., Skinner, D. C., Yahav, S., Moodley, G. P., Cavaleros, M., Zachen, D., Ross, F. P. \& Pettifor, J. M. (1991). Effect of oral cholecalciferol supplementation at physiological and supraphysiological doses in naturally $\mathrm{D}_{3}$-deficient subterranean damara mole rats, Cryptomys damarensis. Journal of Endocrinology 131, 197-202.

Buffenstein, R. \& Yahav, S. (1991a). The effect of diet on microbial population and function in the caecum of the subterranean naked mole rat (Heterocephalus glaber). British Journal of Nutrition 65, 249-258.

Buffenstein, R. \& Yahav, S. (1991 b). Cholecalciferol has no effect on calcium and inorganic phosphorus balance in a naturally cholecalciferol-deplete subterranean mammal, the naked mole rat (Heterocephalus glaber). Journal of Endocrinology 129, 21-26.

Buffenstein, R. \& Yahav, S. $(1991 c)$. Is the naked mole-rat Heterocephalus glaber an endothermic yet poikilothermic mammal? Journal of Thermal Biology 16, 227-232.

Carol, E. J. \& Hungate, R. E. (1954). The magnitude of microbial fermentation in bovine rumen. Applied Microbiology 2, 205-214.

Chertow, B. S., Sivitz, W. I., Baranetsky, N. G., Clark, S. A., Waite, A. \& Deluca, H. F. (1983). Cellular mechanisms of insulin release: The effects of vitamin $D$ deficiency and repletion on rat insulin secretion. Endocrinology 113, 1511-1518.

Clark, S. A., D'ercole, A. J. \& Toverud, S. U. (1986). Somatomedin-C/Insulin like growth factor I and vitamin D-induced growth. Endocrinology 119, 1660-1665.

Clarke, S. A., Stumpf, W. E. \& Sar, M. (1981). Effect of 1,25-dihydroxyvitamin $\mathrm{D}_{3}$ on insulin secretion. Diabetes 30, 382-386.

Davies, J. (1988). Laboratory Methods. Irene, Transvaal: Animal Nutrition Subdirectorate Animal and Dairy Science Research Institute.

El-Din, M. Z. \& El-Shazly, K. (1969). Evaluation of a method of measuring fermentation rates and net growth of rumen micro-organisms. Applied Microbiology 17, 801-804.

Gardener, R. M., Reinhardt, T. A. \& Horst, R. L. (1988). The biological assessment of vitamin $\mathrm{D}_{3}$ metabolites produced by rumen bacteria. Journal of Steroid Biochemistry 29, 185-189.

Jarvis, J. U. M. (1991). Methods for capturing, transporting and maintaining naked mole rats in captivity. In Biology of the Naked Mole Rat, pp. 467-483 [P. W. Sherman, J. U. M. Jarvis and R. D. Alexander, editors]. Princeton: Princeton University Press. 
Jarvis, J. U. M. \& Bennett, N. C. (1991). Ecology and behaviour of the family Bathyergidae. In Biology of the Naked Mole Rat, pp. 66-69 [P. W. Sherman, J. U. M. Jarvis and R. D. Alexander, editors]. Princeton: Princeton University Press.

Kadowaki, S. \& Norman, A. W. (1984). Dietary vitamin D is essential for normal insulin secretion from the perfused rat pancreas. Journal of Clinical Investigation 73, 759-766.

McBee, R. H. (1970). Metabolic contributions of the caecal flora. American Journal of Clinical Nutrition 23, 1514-1518.

Norman, A. W., Frankel, B. J., Heldt, A. M. \& Grodsky, M. (1980). Vitamin D deficiency inhibits pancreatic secretion of insulin. Science 209, 823-825.

Nyomba, B. L., Bouillon, R. \& De Moor, P. (1984). Influence of vitamin D status on insulin secretion and glucose tolerance in the rabbit. Endocrinology 115, 191-197.

Rechkemmer, G., Ronnau, K. \& Engelhardt, W. W. (1988). Fermentation of polysaccharides and absorption of short chain fatty acids in the mammalian hindgut. Comparative Biochemistry and Physiology 90A, 563-568.

Sibly, R. M. (1981). Strategies in digestion and defecation. In Physiological Ecology, and Evolutionary Approach to Resource Use, pp. 109-139 [C. R. Townsend and P. Calow, editors]. Sunderland, Massachusetts: Sinauer Association.

Skinner, D. C., Moodley, G. \& Buffenstein, R. (1991). Is Vitamin $D_{3}$ essential for mineral metabolism in the Damara mole rat (Cryptomys damarensis)? General and Comparative Endocrinology 81, 501-505.

Sommerfeldt, J. L., Horst, R. L., Napoli, J. L., Beitz, D. C. \& Littledike, E. T. (1980). Evidence for in-vitro production of vitamin $\mathrm{D}_{2}$ and vitamin $\mathrm{D}_{3}$ metabolites by rumen microbes. Journal of Dairy Science 63, Suppl. 1, 88-89.

Stumpf, W. E., Sar, M. \& Clark, S. A. (1982). Brain target sites for 1,25-dihydroxyvitamin $\mathrm{D}_{3}$. Science 215 , 1404-1405.

Van Soest, P. J. (editor) (1982). Gastrointestinal fermentation. In Nutritional Ecology of the Ruminants, pp. 152-229. Corvallis, Oregon: O. and B. Books.

Vleck, D. (1981). Burrow structure and foraging costs in the fossorial rodent, Thomomys bottae. Oecologia 49 , 391-396.

Yahav, S. \& Buffenstein, R. (1991). The effect of temperature on caecal fermentation processes in a poikilothermic mammal, Heterocephalus glaber. Journal of Thermal Biology 16, 345-349.

Zar, J. H. (1974). Biostatistical Analysis. Englewood Cliffs, New Jersey: Prentice Hall. 